\title{
Decoding the transcriptome and DNA methylome of human primordial germ cells
}

\author{
HE Chuan \\ Department of Chemistry and Institute for Biophysical Dynamics, The University of Chicago, Chicago, IL 60637, USA; \\ Howard Hughes Medical Institute, Chicago, IL 60637, USA
}

Received June 5, 2015; accepted June 8, 2015; published online June 10, 2015

Citation: He C. Decoding the transcriptome and DNA methylome of human primordial germ cells. Sci China Life Sci, 2015, 58: 729-730, doi: $10.1007 / \mathrm{s} 11427-015-4879-3$

Primordial germ cells (PGCs) are the embryonic founder cells of the gametes- the oocytes and sperms that are vital for transmitting genetic information faithfully and efficiently from one generation to the next and for maintaining the continuation of a species [1]. It is therefore critical to understand the crucial epigenetic processes during the development of this most fascinating lineage. The development of PGCs in mouse as a model organism has been well understood, including their specification, migration, localization to genital ridges, global epigenetic reprogramming, and sex differentiation. However, the mechanistic understanding of gene expression pattern and epigenetic reprogramming in the human PGCs is still largely unknown due to the limited materials and suitable technology available for analysis. By using a single-cell RNA sequencing method and a modified whole-genome bisulfite sequencing technique for limited numbers of cells pioneered by Professor Fuchou Tang and others, Chinese scientists at Peking University have analyzed the transcriptome of human PGCs at migrating and gonadal stages across 15 weeks at the single-cell level and single-base resolution in a recent Cell publication [2]. Their work provides the first comprehensive view of the complex relationship of DNA methylation and gene expression during the global epigenetic reprogramming process of human PGCs.

Similar to mouse PGCs, human mitotic PGCs express pluripotency marker genes such as OCT4, NANOG, and

email: chuanhe@uchicago.edu
REX1. However, human PGCs do not express SOX2, in contrast to murine PGCs. Human PGCs also express germline-specific marker genes, such as KIT, TNAP, AP $2 \gamma$, and NANOS3. It has been shown in a recent publication that SOX17 is a key regulator for human PGC specification in vitro [3]. Tang, Qiao and their collaborator's work [2] extended this finding to show that SOX17 is also expressed in migrating and gonadal PGCs in vivo. More interestingly, SOX15 is expressed much more homogenously and at a significantly higher level in human PGCs than SOX17, particularly at early stages before 10 weeks. This pattern indicates that $S O X 15$ probably plays a more important role in early PGC development in vivo. Although the transcriptome of human PGCs is relatively homogeneous between 4 and 11 weeks, a fraction of the genes show stage-specific expression signatures during PGC development. For example, $T$ and $C D 38$ are expressed in the early PGCs before 7 weeks, whereas VASA, DAZL, TDRD9, and SYCP3 are expressed mainly after 7 weeks. SYCPI and SYCE3 were specifically expressed in meiotic PGCs. Different from the mitotic PGCs between 4- and 11-week, there is a strong gene expression heterogeneity of the 17-week meiotic female PGCs. The 17-week female PGCs in meiosis exhibit a strong heterogeneity of gene expression among individual cells, probably because they are non-synchronized when entering meiosis arrest. Taken together, these unique gene expression signatures indicate that a large set of both pluripotency genes and germline-specific genes are simultaneously expressed within the same individual PGC with a delicate 
balance, which is different from both pluripotent inner cell mass (ICM) in vivo and embryonic stem cells (ESCs) in vitro.

During mouse embryonic development, the inactivated $\mathrm{X}$ chromosome in female PGCs is reactivated during the migration of PGCs into gonads [4]. The total expression level of the $\mathrm{X}$ chromosome-linked genes increased by $\sim 1.6$-fold in 4-week female PGCs compared to those in male PGCs. Moreover, using single-cell RNA sequencing, the authors showed that genes on the $\mathrm{X}$ chromosome exhibit bi-allelic expression in each individual female PGC cells. This result indicates that the inactivated $\mathrm{X}$ chromosome is already reactivated in female PGCs from the migrating stage onward.

Two phases of DNA methylation erasure are known during mouse PGC development: a general phase of global demethylation followed by a more localized phase in which germline-specific genes and imprinted genes are thoroughly demethylated [5]. By using a post-bisulfite adaptor tagging (PBAT) technique for analyzing the DNA methylome of limited number of cells, the global dynamics of DNA methylation in human PGCs could be revealed at between 7-week and 19-week stages. The global DNA methylation level is $91.6 \%$ in the 5-week embryos. However, the methylation level has already decreased to $19.4 \%$ in the 7 -week male PGCs, representing a 4.7-fold decrease, indicating that the major wave of global DNA demethylation occurs in PGCs before 7 weeks of development. The lowest DNA methylation is achieved in the 11-week male and 10-week female PGCs, with only $7.8 \%$ and $6.0 \%$ methylation remaining in the whole genome, representing 12- and 15-fold decreases from early post-implantation embryos, respectively. During this second wave of DNA demethylation, human PGCs display strong enrichment of the DNA base excision repair (BER) pathway related genes, such as $U N G$, $P A R P 1$ and POLB. More interestingly, the DNA dioxygenase TET1 is also highly expressed in PGCs from 4 to 11 weeks, with TET2 and TET3 only mildly expressed. These results suggest that active demethylation probably occurs in human PGCs during this developmental period, similar to that in mouse PGCs at the comparable developmental stages.

Although almost all of the functionally important genomic elements are essentially free of any methylation in 10-11-week human PGCs, such as enhancers, promoters of germline-specific genes, and imprinted DMRs (differential- ly methylated regions). The evolutionarily younger and more active repeat elements, such as L1, Alu, and ERVK, still possess significantly high levels of residual DNA methylation. Similarly, the alpha satellites, which are concentrated in centromeric and peri-centromeric regions, still maintain $\sim 36.5 \%$ DNA methylation. This observation indicates that these repetitive elements are highly methylated even when the global DNA methylation is already minimal in PGCs, which is consistent with the observation made in the mouse, indicating a basis for potential trans-generation inheritance of epigenetic memory.

Although basic features of the transcriptome and DNA methylome landscapes of human migrating and gonadal PGCs are in general similar to those of PGCs at comparable stages in mouse, human PGCs show numerous unique features very different from mouse PGCs. How human PGCs maintain relatively stable global RNA expression patterns when DNA methylation is globally decreased by more than 10 -fold warrants further analysis. This current work represents another major contribution to our understanding of early development events in humans through fruitful collaboration between Professor Fuchou Tang and Professor Jie Qiao. Mechanistic understanding of the complex epigenetic reprogramming of the human germline with the aim of restoring totipotency in fertilized oocytes will be improved by new, transformative discoveries such as this one and more in the future.

1 Surani MA, Durcova-Hills G, Hajkova P, Hayashi K, Tee WW. Germ line, stem cells, and epigenetic reprogramming. Cold Spring Harb Symp Quant Biol, 2008, 73: 9-15

2 Guo F, Yan L, Guo H, Li L, Hu B, Zhao Y, Yong J, Hu Y, Wang X, Wei Y, Wang W, Li R, Yan J, Zhi X, Zhang Y, Jin H, Zhang W, Hou Y, Zhu P, Li J, Zhang L, Liu S, Ren Y, Zhu X, Wen L, Gao Y, Tang F, Qiao J. The Transcriptome and DNA Methylome Landscapes of Human Primordial Germ Cells. Cell, 2015, 161: 1437-1452

3 Irie N, Weinberger L, Tang WW, Kobayashi T, Viukov S, Manor YS Dietmann S, Hanna JH, Surani MA. SOX17 is a critical specifier of human primordial germ cell fate. Cell, 2015, 160: 253-268

4 Chuva de Sousa Lopes SM, Hayashi K, Shovlin TC, Mifsud W, Surani MA, McLaren A. X chromosome activity in mouse XX primordial germ cells. PLoS Genet, 2008, 4: e30

5 Seisenberger S, Andrews S, Krueger F, Arand J, Walter J, Santos F, Popp C, Thienpont B, Dean W, Reik W. The dynamics of genome-wide DNA methylation reprogramming in mouse primordial germ cells. Mol Cell, 2012, 48: 849-862

Open Access This article is distributed under the terms of the Creative Commons Attribution License which permits any use, distribution, and reproduction in any medium, provided the original author(s) and source are credited. 